\title{
Evidence for the Stepwise Behavioral Response Model (SBRM): The effects of Carbamate Pesticides on medaka (Oryzias latipes) in an online monitoring system
}

\author{
Gaosheng Zhang ${ }^{\mathrm{a}}$, Linlin Chen ${ }^{\mathrm{a}}$, Jing Chen ${ }^{\mathrm{a}}$, Zongming Ren ${ }^{\mathrm{a}, \mathrm{b}, *}$, Zijian Wang $^{\mathrm{c}}$, Tae-Soo Chon ${ }^{\mathrm{b}}$ \\ ${ }^{a}$ Key Laboratory of Coastal Zone Environmental Processes, Yantai Institute of Coastal Zone Research, Chinese Academy of Sciences, Yantai 264003, PR China \\ ${ }^{\mathrm{b}}$ Department of Biological Sciences, Pusan National University, Pusan 609735, Republic of Korea \\ 'State Key Laboratory of Environmental Aquatic Chemistry, Research Center for Eco-Environmental Science, Chinese Academy of Science, Beijing 100085, PR China
}

\section{A R T I C L E I N F O}

\section{Article history:}

Received 6 September 2011

Accepted 26 December 2011

Available online 20 January 2012

\section{Keywords:}

The Stepwise Behavioral Response Model

(SBRM)

Medaka (Oryzias latipes)

Carbamate Pesticides (CPs)

Self-Organizing Map (SOM)

Behavior strength (BS)

\begin{abstract}
A B S T R A C T
The Stepwise Behavioral Response Model (SBRM), which is a conceptual model, postulated that an organism displays a time-dependent sequence of compensatory Stepwise Behavioral Response (SBR) during exposure to pollutants above their respective thresholds of resistance. In order to prove the model, in this study, the behavioral responses (BRs) of medaka (Oryzias latipes) in the exposure of Arprocarb (A), Carbofuran (C) and Methomyl (M) were analyzed in an online monitoring system (OMS). The Self-Organizing Map (SOM) was utilized for patterning the obtained behavioral data in $0.1 \mathrm{TU}$ (Toxic Unit), $1 \mathrm{TU}, 2 \mathrm{TU}, 5$ TU, 10 TU and 20 TU treatments with control. Some differences among different Carbamate Pesticides (CPs) were observed in different concentrations and the profiles of behavior strength (BS) on SOM were variable depending upon levels of concentration. The time of the first significant decrease of BS (SD-BS) was in inverse ratio to the $\mathrm{CP}$ concentrations. Movement behavior showed by medaka mainly included No effect, Stimulation, Acclimation, Adjustment (Readjustment) and Toxic effect, which proved SBRM as a time-dependence model based on the time series BS data. Meanwhile, it was found that SBRM showed evident stress-dependence. Therefore, it was concluded that medaka SBR was both stress-dependent and time-dependent, which supported and developed SBRM, and data mining by SOM could be efficiently used to illustrate the behavioral processes and to monitor toxic chemicals in the environment.
\end{abstract}

Crown Copyright $\odot 2011$ Published by Elsevier Ltd. All rights reserved.

\section{Introduction}

Behavioral response (BR) is an important indicator in chemical toxicological impact assessment in all of the test endpoints (Bracy et al., 1978; Farr et al., 1995; Hanazato, 2001; Nakayama et al., 2004; Park et al., 2005; Azizullah et al., 2011). BR to contaminants is an adaptive behavior that may reduce exposure to harmful conditions, but failure to avoid exposure may result in reduced fitness and survival, eventually lead to detrimental effects (Selye, 1973). Every organism has the ability to adapt the environmental stress by intrinsic modification, which might induce evident Stepwise Behavioral Responses (SBRs) including behavior stimulation, behavior acclimation, behavior adjustment and so on (Sun et al., 1993).

The progress of behavioral toxicology, however, was hindered by a lack of test standards as a consensus-based discipline, due to homogeneity of samples, and variation in measured endpoints.

\footnotetext{
* Corresponding author. Address: Key Laboratory of Coastal Zone Environmental Processes, Yantai Institute of Coastal Zone Research, Chinese Academy of Sciences, Chunhui Rd. 18, Laishan District, Yantai 264003, PR China. Tel.: +86 535 2109135; fax: +865352109000.

E-mail address: zmren@yic.ac.cn (Z. Ren).
}

It is essential to provide an objective and comprehensive ground to evaluate behavioral responses. The Stepwise Behavioral Response (SBR) could be a candidate for assessing toxic effects on test organisms through continuous changes of behavioral responses detected by an online monitoring system (OMS).

According to previous research (Selye, 1973; Gerhardt and Janssens de Bisthoven, 2005; Ren et al., 2009a,b), the Stepwise Behavioral Response Model (SBRM) is a conceptual model, postulating that an organism displays a time-dependent sequence of different regulatory or compensatory SBR during exposure to pollutants above their respective thresholds of resistance. When exposed to a pollution, the first response might be avoidance (increased behavioral responses, which meant stimulation), followed by a second response later on under continued exposure, which might be movement acclimation. If these behavior modifications could not adapt the environmental stress based on the respective thresholds of resistance, behavior toxic occurred (Ren et al., 2007).

Carbamate Pesticides (CPs) as widespread development and application, may cause hyperactivity, loss of coordination, convulsions, paralysis and other kinds of behavioral changes of organisms due to ChE inhibition (Casida, 1964; Menn and Mcbain, 1966; Kuhr and Dorough, 1976; Van Dyk and Pletschke, 2011), and then may impair population and biological communities (Shaffer and Gold, 
1985). Medaka (Oryzias latipes) has been reported as a suitable indicator in risk assessment regarding availability of biological information, physiological/behavioral regulation and vulnerability to chemical stress (Gormley and Teather, 2003; Oshima et al., 2003; Chon et al., 2005; Park et al., 2005; Paterson et al., 2011), and it is listed as a standard test species in the OECD guidelines (Organization for Economic Cooperation and Development, 1999).

The aim of the study was to validate and develop (if possible) SBRM based on the behavior effects of Arprocarb (A), Carbofuran (C) and Methomyl (M) on medaka in an OMS, as extensive use and discharges of CPs in environment is not likely to change in the foreseeable future. Behavioral data were trained by SelfOrganizing Map (SOM) to differentiate behavioral responses based on behavior strength (BS). Experiments with continuous treatment along with computational methods were illustrated in objectively characterizing complex behavioral data in response to different chemicals and concentrations.

\section{Materials and methods}

\subsection{Equipment}

BR of medaka was monitored by OMS built in Chinese Academy of Sciences (Li et al., 2007). Test organisms were placed in a flowthrough test chamber ( $7 \mathrm{~cm}$ long, $5 \mathrm{~cm}$ in diameter), which is closed off on both sides with nylon nets $(250 \mu \mathrm{m})$. One pair of electrodes at the walls of the test chambers sends a high frequency signal of altering current, which is received by a second pair of non current-carrying electrodes (Wang et al., 2007). BS of test organisms is transformed by the A/D transformer and the signal changes formed by the A/D transformer were analyzed automatically by software attached to the equipment (Fig. 1). BS is sampled automatically by OMS every second, and BS average value every $6 \mathrm{~min}$ are used to analyze behavioral changes by comparing sample values in OMS control database. BS that changed from 0 (Lose the ability of movement) to 1 (Full behavior express) was introduced to illustrate the BR differences of medaka. The judgment standard of significant decrease of BS (SD-BS) was that: the around difference of BS ( $30 \mathrm{~min}$ ) mean value changed no less than 20\% (Ren et al., 2009a).

\subsection{Test species}

The individuals of medaka fish were kindly provided by the Research Center for Eco-Environmental Sciences, Chinese Academy of Sciences (Beijing, China). The brood stock was raised in flowthrough system with dechlorinated tap water (using active carbon) at a constant temperature of $20 \pm 2{ }^{\circ} \mathrm{C}$, and has been maintained in our laboratory for more than three years. Control the culturing photoperiod of $16 \mathrm{~h}$ light (04:00-20:00, with 4000 lux light intensity) and 8 h dark (20:00-04:00, no light given). The brood stock was fed with newly hatched brine shrimp in the morning and flake food (Trea ${ }^{\circledR}$, Germany) in the afternoon. By $15 \mathrm{~d}$ later after hatching, medaka was fed two times every day. Medaka individuals ( 2 months) about $2.5 \sim 3.0 \mathrm{~cm}$ long were selected as the test organisms.

\subsection{Test chemicals}

Arprocarb (A), Carbofuran (C) and Methomyl (M) were purchased from J\&K Chemical Ltd. All compounds were technical grade ( $>95 \%$ purity). Stock solutions (stored at $4{ }^{\circ} \mathrm{C}$ until use) with proper concentration of each chemical were prepared in dimethyl sulfoxide (DMSO) with appropriate aliquots used to make each test solution of a specific concentration. All solvents were of analytical grade. The concentration of DMSO was less than $0.5 \%$ in all experiments, which would neither lead to acute toxicity to medaka nor affect the mobility (Sandbacka et al., 2000).

\subsection{Experimental setup}

BR monitoring was carried out under flow-through conditions. Three healthy medaka ( 2 months young) about $2.5 \sim 3.0 \mathrm{~cm}$ long were selected at random for each test chamber. Controlled the flow

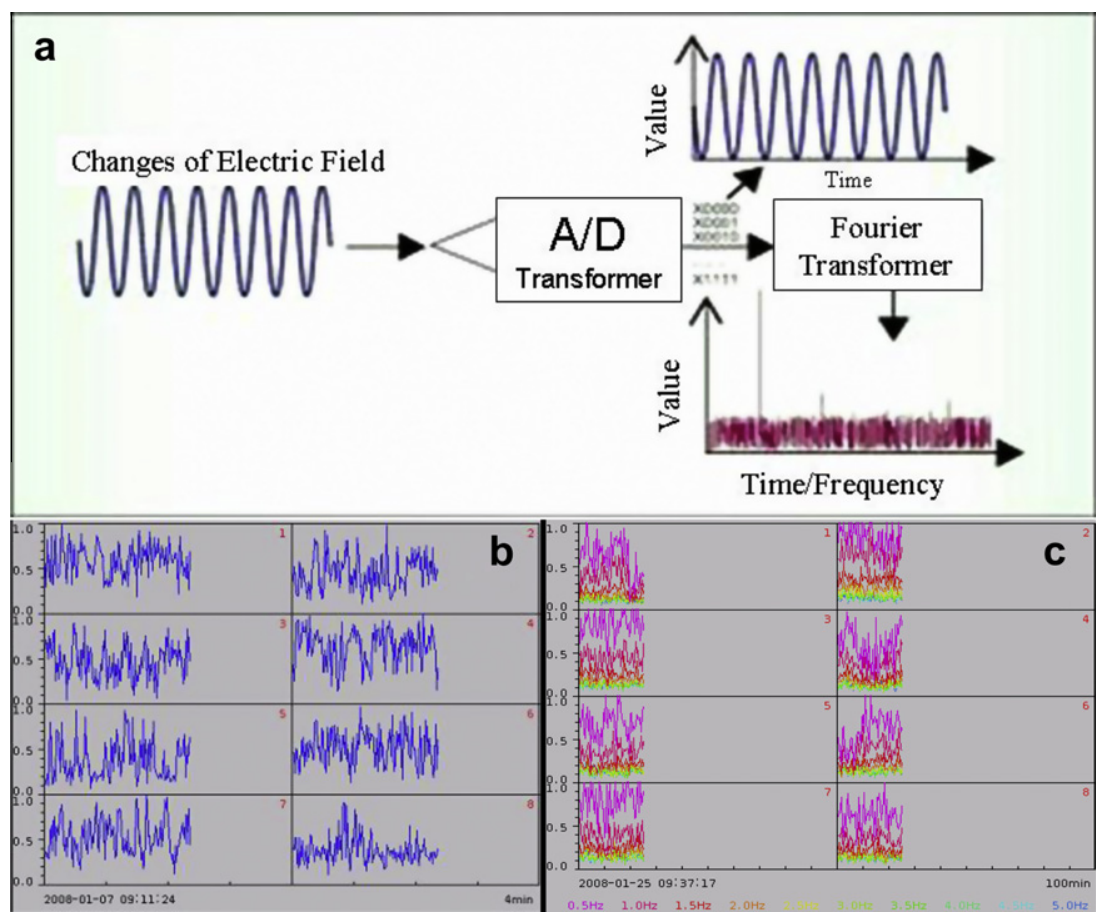

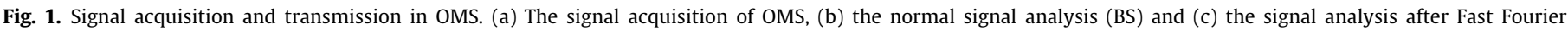
Transform. 
rate of each test chamber about $2 \mathrm{~L}$ per hour, which was proved to have no effect on the motility of test organisms (Guilhermino et al., 2000). During these experiments, no food was added. Control the experiment photoperiod of $16 \mathrm{~h}$ light (04:00-20:00) and $8 \mathrm{~h}$ dark (20:00-04:00).

A 48-h exposure experiment to A, C and M respectively were adopted to investigate medaka SBR. Observation started in 16:00, and finished in 16:00 of the 3rd d for all chambers. Based on the acute toxic results of the three CPs on medaka, the 48-h median Lethal Concentration ( $\mathrm{LC}_{50-48}$ ) was $10.0 \mathrm{mg} \mathrm{L}^{-1}, 0.87 \mathrm{mg} \mathrm{L}^{-1}$, $1.5 \mathrm{mg} \mathrm{L}^{-1}$ for $\mathrm{A}, \mathrm{C}$ and $\mathrm{M}$ respectively. Chemical Toxic Unit (TU) to test organisms was used for comparison, i.e., the $\mathrm{LC}_{50-48}$ was taken as one unit ( $1 \mathrm{TU})$. Sublethal concentration was regarded as $10 \% \mathrm{LC}_{50}$ or less (Kong et al., 2000). Therefore, the 48 -h exposure tests under flow-through conditions by online chemical-mix equipment were performed for 6 concentration gradients, i.e., $0.1 \mathrm{TU}, 1 \mathrm{TU}, 2 \mathrm{TU}, 5 \mathrm{TU}, 10 \mathrm{TU}$ and $20 \mathrm{TU}$ respectively, and repeated thrice for each 48-h exposure comparing with control. The calculation of TU value was as follows (Kong et al., 2000):

$T U=\sum \frac{C_{i}}{\mathrm{LC}_{50}^{i}}$

where $C_{i}$ was the total concentration of chemicals, $\mathrm{LC}_{50}^{i}$ was the 48-h median Lethal Concentration.

All polluted water used in this study was treated with active carbon after exposure.

\subsection{Data analysis}

Output data from OMS were analyzed with SOM to provide overall patterns of behavior in response to chemicals. Based on the solutions presented in Rabiner (1989), the process was conducted with the programs provided in the HMM toolbox (MATLAB7.8, The Math Works, R2009).

SOM performs a non-linear projection of data onto a space in two dimensions and provides a patterned map of input data trained with unsupervised learning (Kohonen, 2001). The size of the SOM was determined heuristically in such a way that could be comprehensible to the reader in a smaller number of dimensions. The highest variance in the input data will be projected along with the vertical axis while the following variance would be accordingly presented on the horizontal axis. Optimal size of computational modes was adjusted based on degree of discrimination among the grouped nodes after training. About two thirds of the total nodes were occupied by the nodes with the samples, while one third was empty nodes, which served as delimiters between the occupied nodes. Through preliminary training, the size of $16 \times 16$ nodes was used in this study.

The Euclidian distance $\left(d_{j}(t)\right)$ at the $j$-th node on the SOM between weight at iteration time $t$ and the input vector was calculated through learning processes:

$d_{j}(t)=\sum_{i=0}^{P-1}\left[x_{i}-w_{i j}(t)\right]^{2}$

where $x_{i}$ is the value of the $i$-th parameter, $w_{i j}(t)$ is the weight between $i$-th parameter and the $j$-th node on the SOM and $P$ is the number of the parameter.

The best matching neuron, which has the minimum distance, was chosen as the winner. For the best matching neuron and its neighborhood neurons, the new weight vectors are updated as:

$w_{i j}(t+1)=w_{i j}(t)+a(t)\left[x(t)-w_{i j}(t)\right]$

where $t$ is the iteration time and $\alpha(t)$ is the learning rate. The learning process of the SOM was conducted using the SOM Toolbox developed by the Laboratory of Information and Computer Science,
Helsinki University of Technology in Matlab environments (Vesanto et al., 2000). The initialization and training processes followed suggestions by the SOM Toolbox by allowing optimization in algorithm. Detailed description regarding application of the SOM to behavioral data can be referred to other relative reports (Park et al., 2005).

As the input data were provided to the SOM for training (Eq. (3)), the weights of the best matching unit and computation nodes close to it were adjusted towards the input vector through interactive calculation. In order to reveal the degree of association between the SOM units, the Ward's linkage method was used to cluster the movement data based on the dendrogram according to the Euclidean distance (Ward, 1963; Wishart, 1969). The linkage distances were rescaled in $0-100 \%$.

\section{Results and discussion}

\section{1. $C P$ effects on medaka $B R$}

After SOM, medaka BR was shown according to different CPs with control (Fig. 2). Exposure time was in accordance with photoperiod (Fig. 2a). According to cluster analysis, six groups were identified based on the mean values of BS (Fig. 2b and c). Cluster 4 at the bottom left corner of SOM presented initial period of $\mathrm{CP}$ treatment, and cluster 6, 5, 2 and 1 reflected subsequent exposure time. Cluster 3 was empty nodes, which served as delimiters between the occupied nodes, such as cluster 1, 2, 4, 5 and 6 . The cluster distances according to the Ward's linkage method indirectly suggested closeness between clustered groups (Fig. 2c).

Fig. 2d shows the profiles of BS values visualized on the SOM in different CPs. The values in the vertical bar indicate the range of BS mean values according to different concentrations and different time period. In the control group, BS ranged between 0.693 and 0.893 , and higher values were observed at the start time (cluster 4 and 6) and mid time (cluster 2) based on the results of SOM (Fig. 2d). Degree of changes was relatively lower than that by treatments. By matching with Fig. 2c, BS changes of medaka showed an evident rhythm (Binkley et al., 1978): higher BS value matched to the light photoperiod along with diagonal line from bottom-left to upper-right, while the lower values were in accordance with area of SOM in the dark period (cluster 5). At the end of the exposure at upper-left corner, though it was in photophase, BS values were lower than the area long with diagonal line due to no food supplied in $48 \mathrm{~h}$. While some differences among different CPs gradient was observed in different concentrations. The profiles of BS on SOM were variable depending upon levels of concentration. At lower levels, profiles were different according to different CPs, but the values were lower, and even sublethal concentration could induce behavior toxic effects, which were reflected by the disorder of the internal rhythm caused by biological clock showed in control. Significant BS decrease happened in cluster 1 and 2 in 1 TU and 2 TU exposure, cluster 2 and 5 in 5 TU exposure, and cluster 6 in 10 TU and 20 TU. Meanwhile, medaka BS dropped to less than 0.1 soon after the start of $10 \mathrm{TU}$ and $20 \mathrm{TU}$ exposures. In all these higher level exposures, no more circadian rhythms were observed. These results suggested that medaka BR based on BS was time-dependent in the same exposure level.

Fig. 3 shows the exposure time of medaka in different CPs before the first SD-BS. Though the difference of individual SD-BS was great based on the analysis of the standard deviation, the total tendency was the same. The results suggested that SD-BS was strongly depended on exposure concentrations. The first SD-BS kept similar at the same concentrations in different CPs.

After the first SD-BS, BR of medaka were different in different concentrations: in $10 \mathrm{TU}$ and $20 \mathrm{TU}$, BS dropped suddenly to less than 0.1 , which suggested that test medaka might lose movement; 
a 46

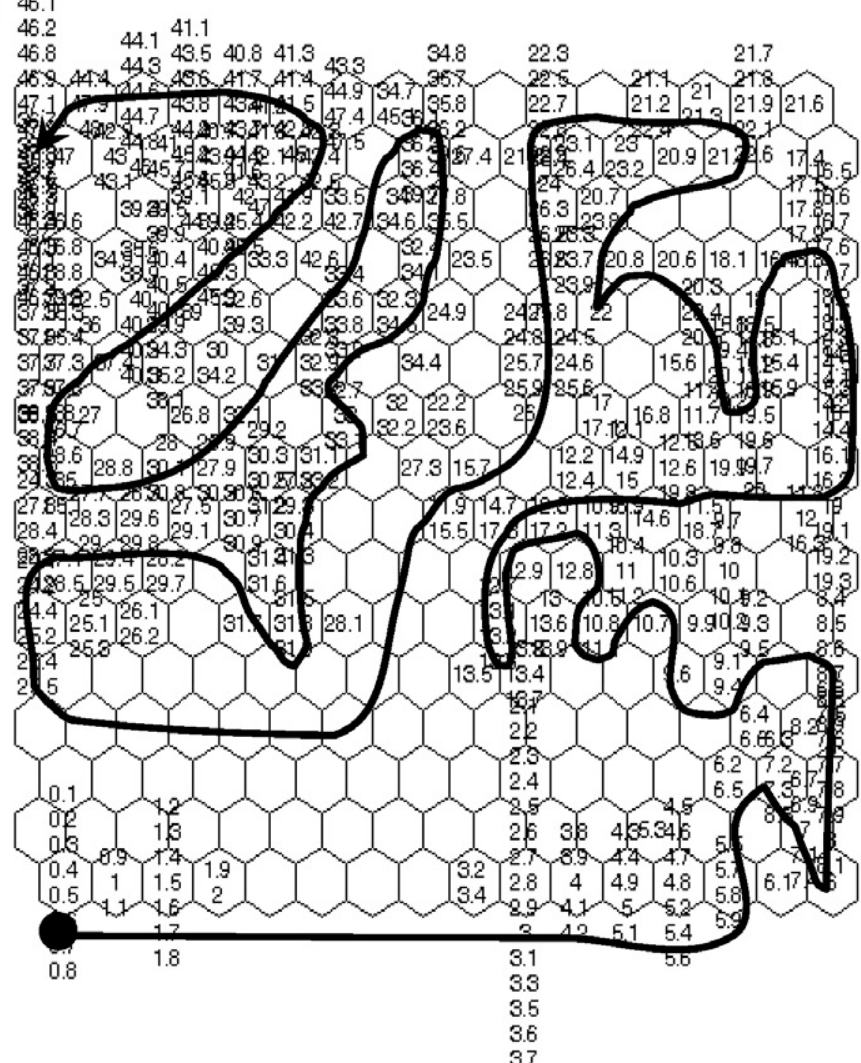

b

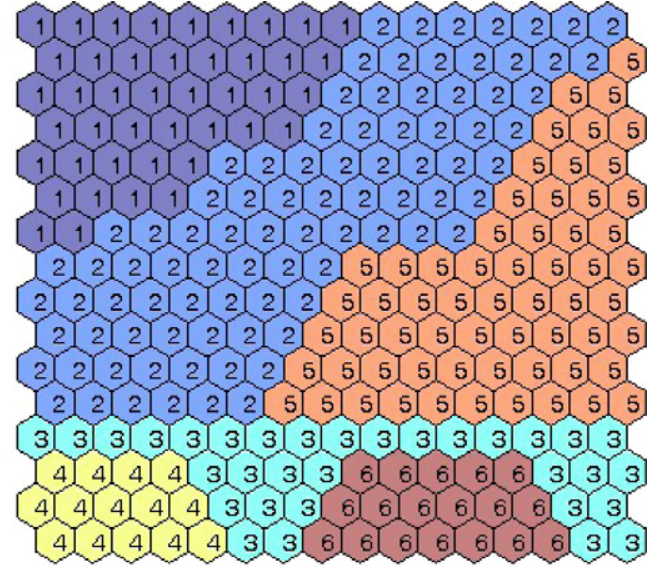

c

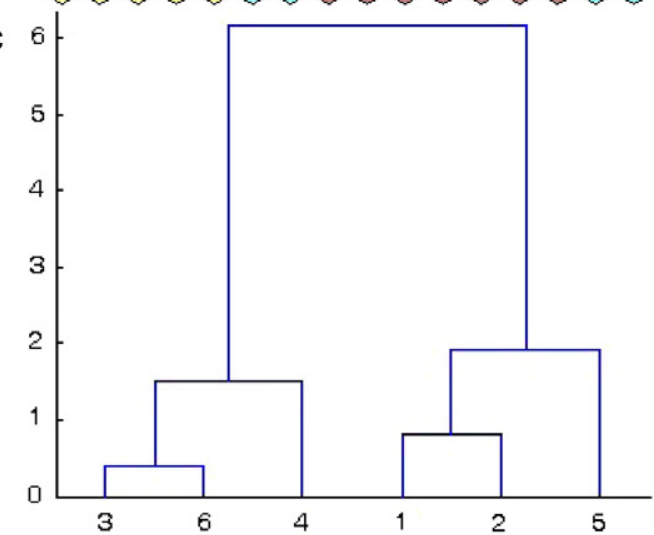

A2TU
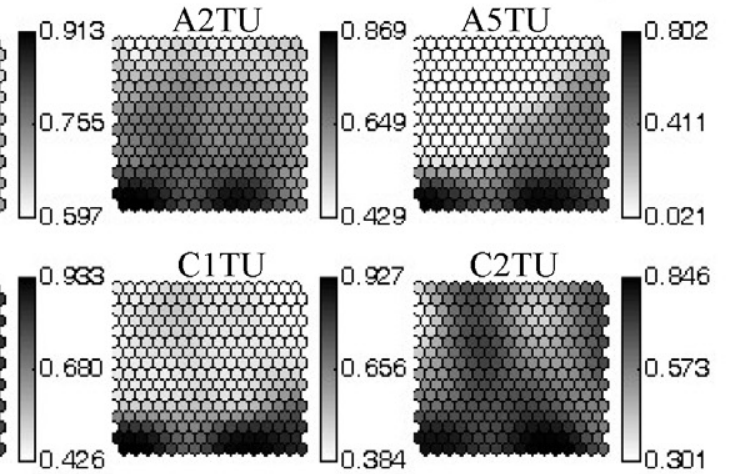
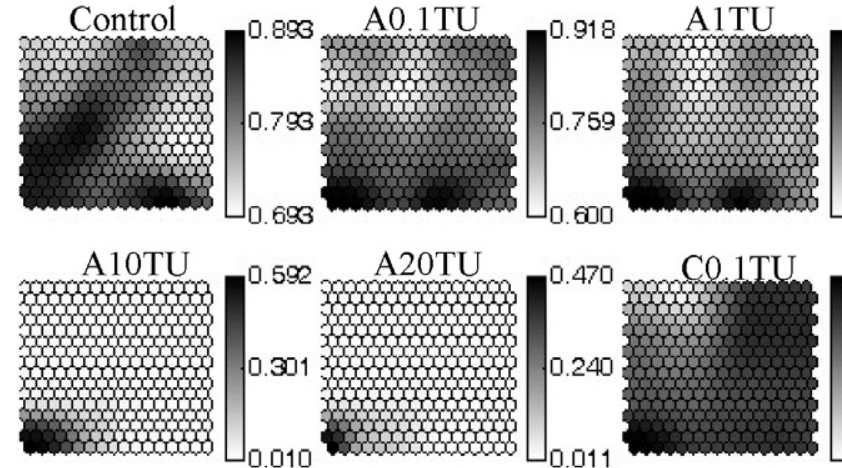

A20TU
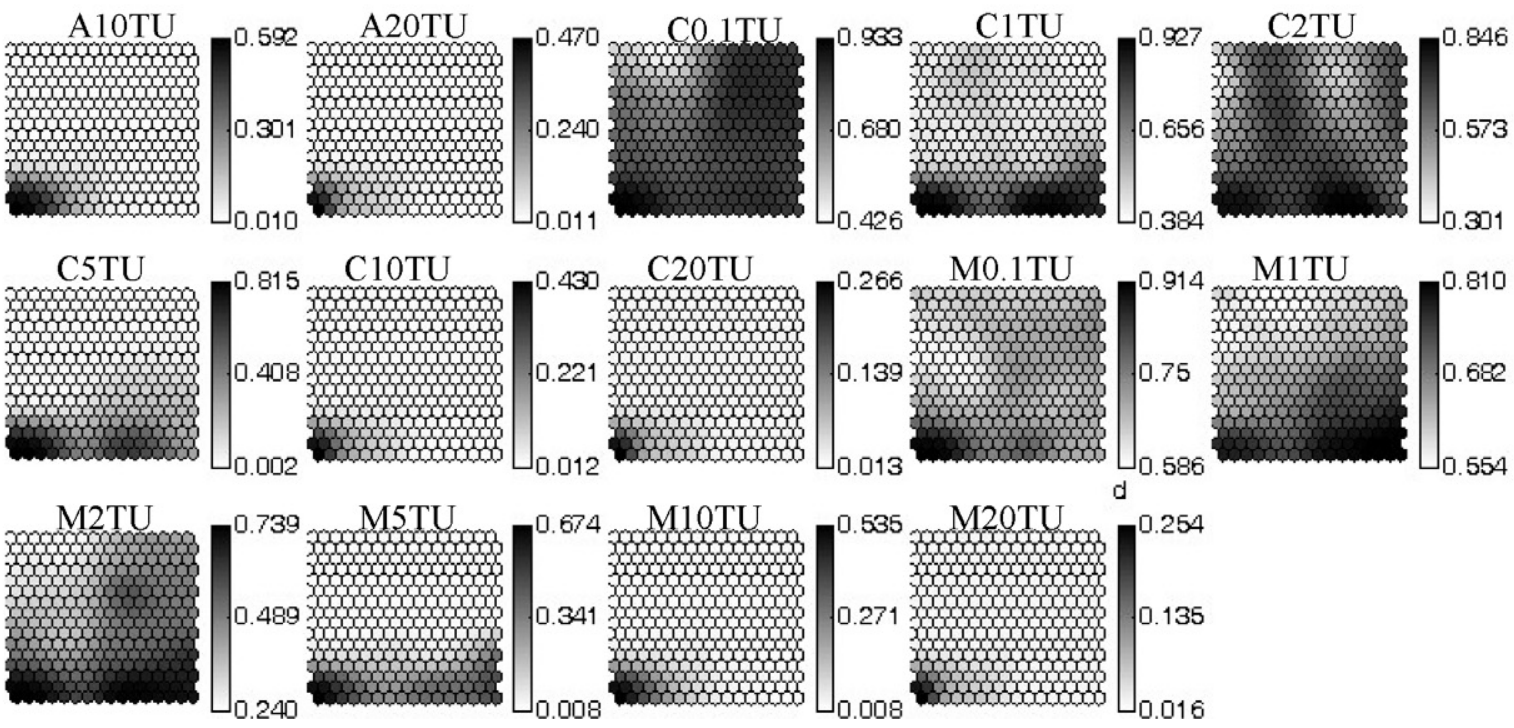

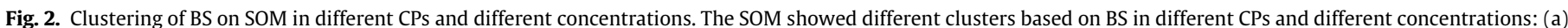

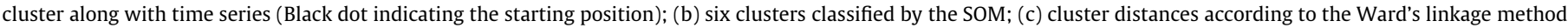

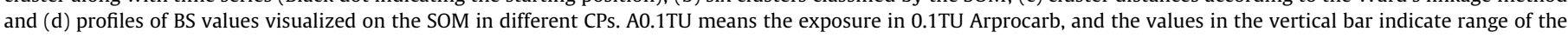
mean values of BS.

however, in $5 \mathrm{TU}, 2 \mathrm{TU}, 1 \mathrm{TU}$ and $0.1 \mathrm{TU}$, the significant stepwise modulation appeared, and the modulation movements included acclimation, adjustment and readjustment. Once, the movement regulation could not satisfy the intrinsic response requirement to environmental stress ( $2 \mathrm{TU}$ and $5 \mathrm{TU}$ ), significant toxic effects would happen as shown in Fig. 2. Therefore, it was concluded that 


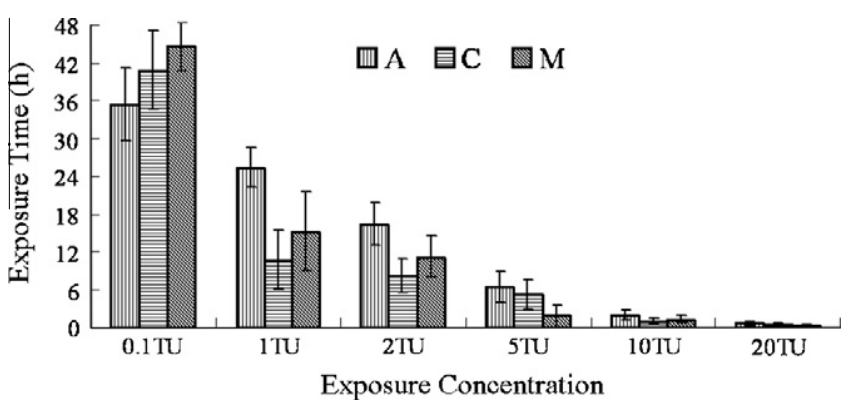

Fig. 3. The exposure time of medaka in different concentration CPs before the first SD-BS. *Showed in $\mathrm{M} \pm$ S.D.

SBR was very important for medaka adaptation to the environmental stress, especially in lower concentrations. Meanwhile, these results advised that medaka SBR (or BR) was stress-dependent.

\subsection{Medaka SBR}

The effects of $\mathrm{A}, \mathrm{C}$ and $\mathrm{M}$ on medaka BR in different exposure time were showed in Fig. 4. Medaka BS in the control kept about 0.8 , which was similar to the start period of every exposure (the first $6 \mathrm{~min}$ ). These results illustrated that it showed almost no negative effects on the behavioral responses both in the control and at the start period (the first $6 \mathrm{~min}$ ) of different treatments. In treatments of the same chemical, the concentration and the exposure time affected the BR evidently, which showed a stepwise change. It advised that the higher concentration and longer exposure time could induce more evident decrease of BR. The same tendency happened in every treatment, which suggested that different concentration and different exposure time induced different BS.

In different chemical treatments, the results suggested that the tendency of medaka BR in the exposure of different CPs was similar. All the BR tendencies in different treatments were decrease. The BR in these proceeds did not show smooth decrease change especially in lower concentrations, but with several adjustments/ readjustment, which advised that $B R$ was stepwise modulation during CPs exposure in treatment.

Based on the effects of CPs on medaka SBR in time series and stress series (Fig. 5), medaka BR showed evident time-dependent decrease, followed by a second and a third increase in lower concentrations (Fig. 5a). Behavior remained influenced by circadian rhythms in control and lower concentration CP stress. But in treatments, CPs might have an increasing effect on medaka BS as well as on circadian rhythm. The latter was related to endocrine disrupting action of CPs on melatonin and serotonin physiology. Such loss of rhythmicity has also been observed in the fish Anabas testudineus exposed to the insecticide lindane (Bakthavathsalam et al., 1987).

In lower concentration treatments, e.g. $0.1 \mathrm{TU}$ and $1 \mathrm{TU}$, medaka SBR was more significant, which mainly contained Stimulation, Acclimation, and Adjustment (Readjustment), without Toxic effect. In 5 TU CP treatments during the exposure period, medaka BR met a great many changes, which mainly included Stimulation, Acclimation, Adjustment (Readjustment) and Toxic effect (Ren et al., 2007). In the highest concentration exposure (10 TU and $20 \mathrm{TU}$ ), it was hard for medaka to have the capability of adjustment. Medaka BR decreased with hardly any movement modulation (Fig. 5a), and the reason for this might be that in the higher concentration exposure, the intrinsic responses were not strong enough to adjust inner environment by BR to adapt the outside stress (Sun et al., 1993). In stress series data analysis as shown in Fig. 5b, the similar BR tendency was observed though selected data were not abundant as of time series.
Based on these results, medaka SBR was illustrated clearly by the tendency curves $(\alpha, \beta, \gamma$, and $\delta$ ) both in time series and stress series data analysis. Higher toxic effects on behavior movement induced BS decrease as curve $\alpha$ with hardly any adjustment, and in lower environmental stress, BR tendency could be reflected by curve $\gamma$ and $\delta$, which included behavior stimulation, acclimation, adjustment and toxic effects as described by Selye (1973). Behavior stimulation was usually followed by acclimation which was a continuous weakening of BS. The main reason for the fall of movement behavior in acclimation was that the behavior adjustment reached extreme of "Alarm reaction" due to "threshold (I, II, and III)" showed as the curve inflection points (1, 2, and 3) in Fig. 5.

The effects of CPs on medaka SBR suggested that different environmental stress in different exposure period decided medaka BR. Therefore, it was concluded that medaka SBR was affected by both exposure time and exposure concentrations, and higher environmental stress might limit the function of behavior adjustment caused by intrinsic responses mechanisms.

\subsection{Stepwise Behavioral Response Model (SBRM)}

The results of medaka SBR based on both time series and stress series suggested that the behavior movements of medaka in different CP treatments went through No effect, Stimulation, Acclimation, Adjustment (Readjustment) and/or Toxic effect (Fig. 5). These results were accordance with previous studies on Daphnia magna (Ren et al., 2007, 2009a; Ren and Wang, 2010), which advised that in organophosphorous pesticide exposure, a cascade of regulatory behavioral stress responses were activated and performed by the organisms. Meanwhile, the results in this study proved that either increasing toxicant concentration or exposure time, a cascade of regulatory behavioral stress responses were activated and performed by the organisms. Therefore, medaka SBRM postulated that an organism displayed both stress-dependent and time-dependent of different regulatory or compensatory behavioral stress responses during exposure to pollutants above their respective thresholds of resistance (Fig. 6). An increasing stress stimulus provoked regulatory responses (loading stress). Above a certain stimulus level, however, several reactions were possible: (1) the homeostasis could not be maintained and a toxic effect in the organism occurs (limiting stress), (2) the organism could acclimate to the increased stress level and (3) the organism decreased the performance of the response and increased the performance of another response to the stimulus. If the first stress response decreased to less than the original level, a toxic effect occurs.

In theory, the first behavior modulation of medaka was to increase the strength of all frequency movements presumably to try to escape from the polluted aquatic environment (Avoidance behavior) (Ren et al., 2009a), which explained the BR observed in Stimulation after No effect. Presumably, stress of certain CP concentrations would be too high for medaka to start Avoidance behavior because this type of behavior was absent or of short duration at high CP concentrations (Figs. 4 and 5a). In these cases, BS tended to decrease gradually until the ability for movement was lost. In other cases, medaka was apparently able to cope with the neurotoxin stressors and increased BS. Associated with this BR, the length of Stimulation was close to the expected value, about 1-3 h (Fig. 3) and it was in inverse ratio to the CP concentrations.

Stimulation was usually followed by Acclimation which was a continuous weakening of BS. The main reason for the fall of movement behavior in Acclimation was that the behavior adjustment reached extreme of "Alarm reaction" (Threshold 1, showed as thick vertical line I in Fig. 5). After Acclimation, BR split in two directions: First, if medaka could not overcome Threshold 1, Toxic effect would occur. Secondly, if medaka lived through Threshold 1, BS resumed in a short time as shown in Adjustment and there would be a second 

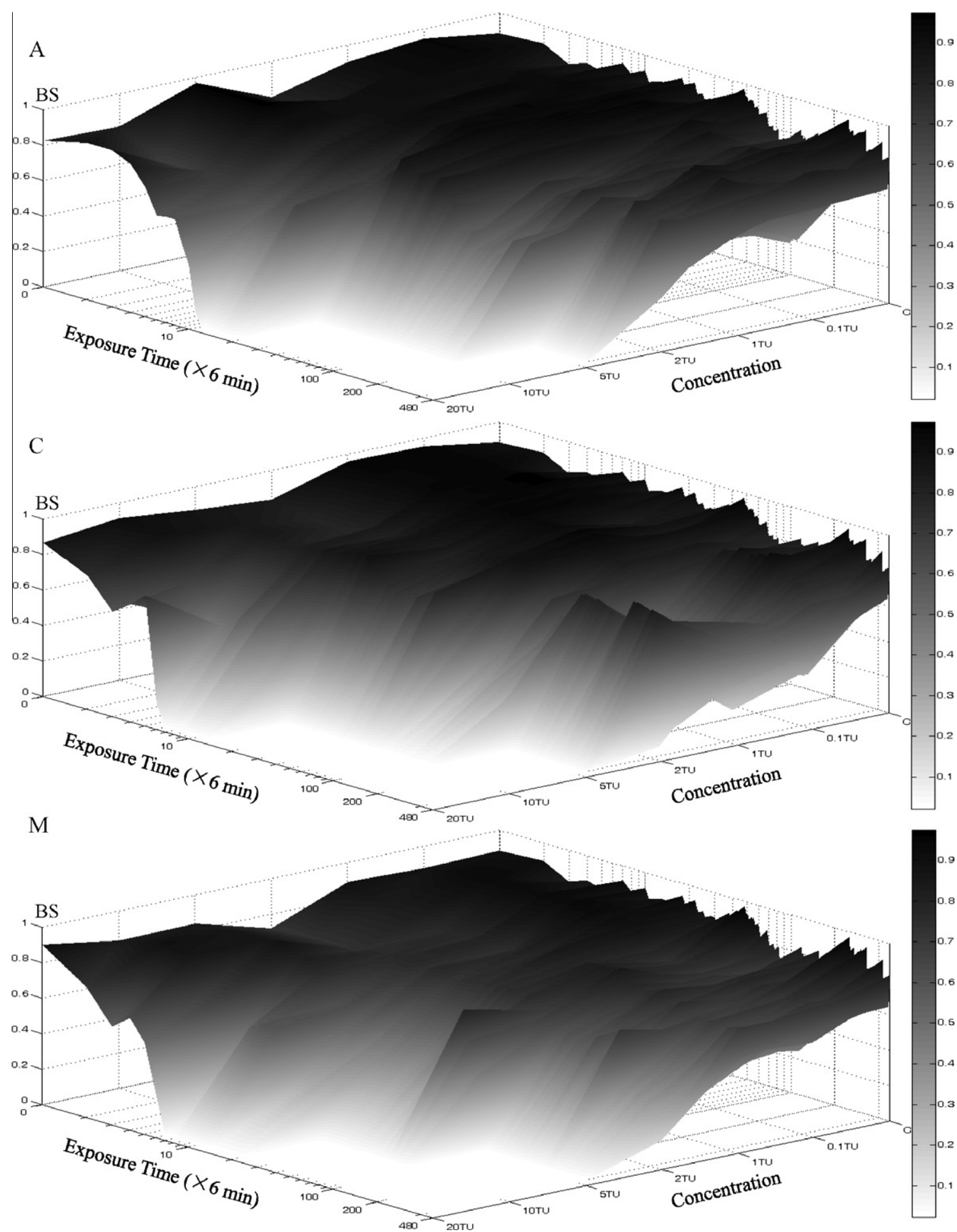

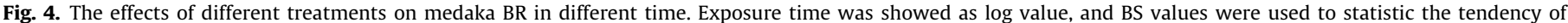
medaka BR in different CPs. A, C and M meant the effects of Arprocarb, Carbofuran and Methomyl separately on medaka BR.

or a third extreme of "Alarm reaction" (Threshold 2 or 3 , showed as thick vertical line II and III in Fig. 5) until Toxic effect occurred.

Based on these results, "avoidance behavior" was quite appropriate to demonstrate SBR as reported in previous research (Ren et al., 2009b), as almost all aquatic animals have the ability of actively escaping from a polluted environment to an unpolluted area (Robin and Don, 1971). According to medaka SBRM, a trend for behavior modulation to maintain a stable internal environment and diminish dependence on an external environment was also shown (Putman and Wratten, 1984).

These results that medaka SBR was both stress-dependent and time-dependent, which supported and developed SBRM according to previous researches (Selye, 1973, Gerhardt and Janssens de Bisthoven, 2005; Ren et al., 2007, 2009a). Meanwhile, it was "Threshold" that advised the effects of environmental stress on BR of medaka and decided the tendency of BR. 

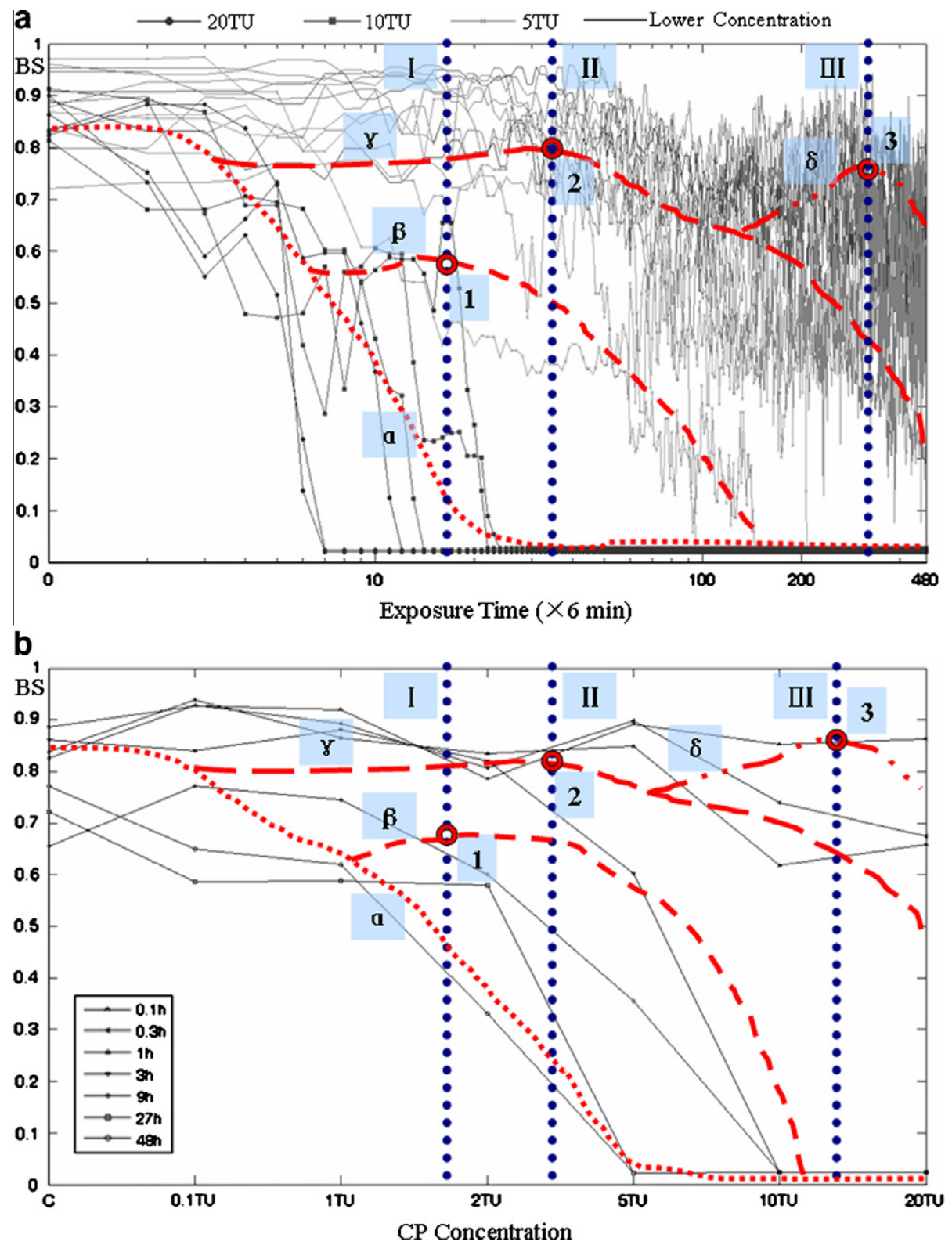

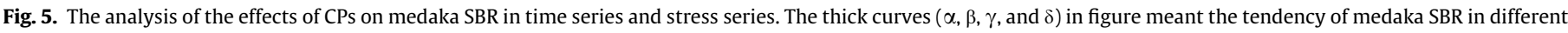

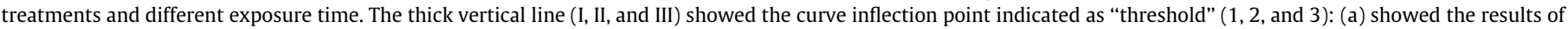

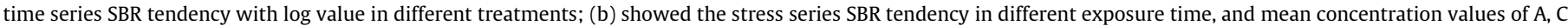

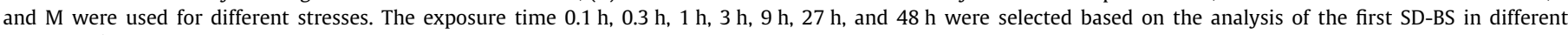
concentrations.

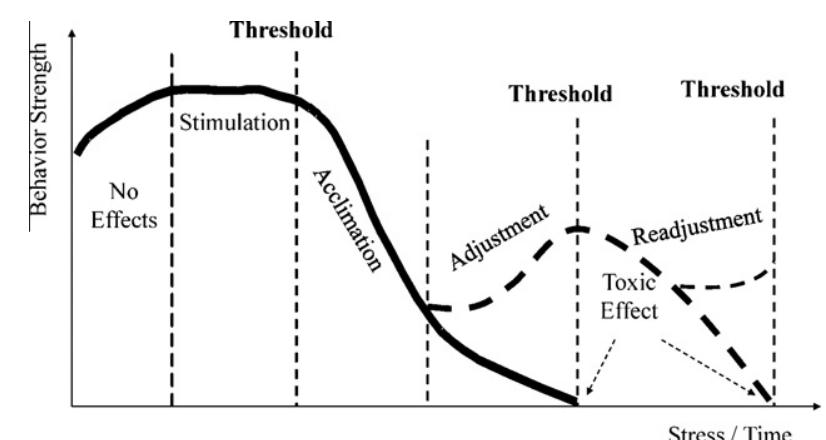

Fig. 6. SBRM of medaka in CP exposure.

\section{Conclusions}

As the inhibitor of ChE, the toxicity characteristics of all CPs on the behavior movement of organisms were the same (Menn and Mcbain, 1966). The activity restrain of ChE resulted in unregulated nerve ending activation and paralysis in organisms (Casida, 1964), which could induce the loss of the nerve conduction ability, and then cause hyperactivity, loss of coordination, convulsions, paralysis and other kinds of behavioral changes. All of these behavior disorders could bring on SBR of organisms. Therefore, the SBR could be used as a suitable indicator in eco-toxicological risk assessment of CPs. Behavioral states obtained by using the SOM were accordingly classified in a heuristic manner, and subsequently, the movement strength data were verified with the SBR processes under stressful conditions. Data mining by SOM and SBR processes 
in combination could be efficiently used to illustrate the behavioral processes and to monitor toxic chemicals in the environment.

The both stress-dependent and time-dependent SBRM in this study proved and developed the previous research results. Whereas, it is necessary to do more experiments on the effects of more test organisms exposed to more chemicals to verify the behavior model. Meanwhile, since it might be the activity restrain of ChE that resulted in SBR of medaka (Xuereb et al., 2009), further investigation on the effects of CPs on medaka should focus on the relationship between the restrain degree of $\mathrm{ChE}$ and the BR by in vivo test to discuss the intrinsic response mechanism of the stepwise model.

\section{Acknowledgments}

This study was financially supported by the National Natural Science Foundation of China (21107135) and the 2011 Post-Doc. Development Program of Pusan National University of Korea.

\section{References}

Azizullah, A., Richter, P., Häder, D.P., 2011. Comparative toxicity of the pesticides carbofuran and malathion to the freshwater flagellate Euglena gracilis. Ecotoxicology 20 (6), 1442-1454.

Bakthavathsalam, R., Balasubramanian, U., Ravikumar, G., 1987. Timedependent effects of lindane (ç-BHC) on the bimodal oxygen consumption of Anabas testudineus (Bloch). Pesti. Biochem. Physiol. 28, 318-324.

Binkley, S.A., Riebman, J.B., Reilly, K.B., 1978. The pineal gland: a biological clock in vitro. Science 202. 1198-1120.

Bracy, O.L., Doyle, R.S., Kennedy, M., Mcnally, S.M., Weed, J.D., Thorne, B.M., 1978. Effects of methomyl and ethanol on behavior in the sprague-dawley rat Pharmacol. Biochem. Be. 10, 21-25.

Casida, J.E., 1964. Esterase inhibitors as pesticides. Science, 1011-1017.

Chon, C.N., Kwak, I., Kim, J., Koh, S., Lee, S., Leem, J., 2005. Movement behavior of medaka (Oryzias latipes) in response to sublethal treatment of diazinon and cholinesterase activity in semi-natural conditions. Environ. Monit. Assess. 101, $1-21$.

Farr, A.J., Chabot, C.C.T., Douglas, H., 1995. Behavioral avoidance of fluoranthene by fathead minnows (Pimephales promelas). Neurotoxicol. Teratol. 17, 265-271.

Gerhardt, A., Janssens de Bisthoven, L., 2005. Evidence for the stepwise stress model: gambusia holbrooki and daphnia magna under AMD and ACID stress. Environ. Sci. Technol. 39 (11), 4150-4158.

Gormley, K.L., Teather, K.L., 2003. Developmental, behavioral, and reproductive effects experienced by Japanese medaka (Oryzias latipes) in response to shortterm exposure to endosulfan. Ecotox. Environ. Safe. 54, 330-338.

Guilhermino, L., Diamantino, T., Silva, M.C., Soares, A.M.V.M., 2000. Acute toxicity test with daphnia magna: an alternative to mammals in the prescreening of chemical toxicity. Ecotox. Environ. Safe. 46, 357-362.

Hanazato, T., 2001. Pesticide effects on freshwater zooplankton: an ecological perspective. Environ. Pollut. 112, 1-10.

Kohonen, T., 2001. Self-Organizing Maps, Third Extended Edition. Springer, Berlin.

Kong, F., Yin, D., Yan, G., 2000. Environmental Biology. Higher Education Press, Beijing (In Chinese).

Kuhr, R.J., Dorough, H.W., 1976. Mode of action. In: Carbamate Insecticides: Chemistry, Biochemistry, and Toxicology. CRC, Cleveland, OH, pp. 41-70.
Li, Z., Ren, Z., Ma, M., Zha, J., Wang, Z., Fu, R., 2007. Research on the behavioral responses of Daphnia magna in the early warning of organophosphorous pesticide contamination. China. Water. Wastewater. 23, 73-75.

Menn, J.J., Mcbain, J.B., 1966. Detection of cholinesterase-inhibiting insecticide chemicals and pharmaceutical alkaloids on thin-layer chromatograms. Nature 209, 1351-1352.

Nakayama, K., Oshima, Y., Yamaguchi, T., Tsuruda, Y., Kang, I.J., Kobayashi, M., Imada, N., Honjo, T., 2004. Fertilization success and sexual behavior in male medaka, Oryzias latipes, exposed to tributyltin. Chemosphere 55 (10), 13311337.

Organization for Economic Cooperation and Development, 1999. Final Report of the Fish Expert Consultation Meeting, London, UK, October, 28-29, 1998; Paris, Cedex 16, France.

Oshima, Y., Kang, I., Kobayashi, M., Nakayama, K., Imada, N., Honjo, T., 2003. Suppression of sexual behavior in male Japanese medaka (Oryzias latipes) exposed to 17b-estradiol. Chemosphere 50, 429-436.

Park, Y., Chung, N., Choi, K., Cha, E., Lee, S., Chon, T., 2005. Computational characterization of behavioral response of medaka (Oryzias latipes) treated with diazinon. Aquat. Toxicol. 71, 215-228.

Paterson, G., Ataria, J.M., Hoque, M.E., Burns, D.C., Metcalfe, C.D., 2011. The toxicity of titanium dioxide nanopowder to early life stages of the Japanese medaka (Oryzias latipes). Chemosphere 82 (7), 1002-1009.

Putman, R.J., Wratten, S.D., 1984. Principles of Ecology. Groom Helm, London, Canberra.

Rabiner, L.R., 1989. A tutorial on hidden Markov models and selected applications in speech recognition. Proc. IEEE 77 (2), 257-286.

Ren, Z., Li, Z., Zha, J., Ma, M., Wang, Z., Fu, R., 2009a. Behavioral responses of Daphnia magna to stresses of contaminants with different toxic mechanisms. B. Environ. Contam. Tox. 82, 310-316.

Ren, Z., Li, Z., Zha, J., Ma, M., Wang, Z., Gerhardt, A., 2007. The early warning of aquatic organophosphorus pesticide contamination by on-line monitoring behavioral changes of Daphnia magna. Environ. Monit. Assess. 134, 373-383.

Ren, Z., Li, Z., Zha, J., Rao, K., Ma, M., Wang, Z., Fu, R., 2009b. The avoidance responses of Daphnia magna to the exposure of organophosphorus pesticides in an on-line biomonitoring system. Environ. Model. Assess. 14, 405-410.

Ren, Z., Wang, Z., 2010. The differences in the behavior characteristics between Daphnia magna and Japanese madaka in an on-line biomonitoring system. J. Environ. Sci. 22 (5), 703-708.

Robin, H., Don, R., 1971. Shrimps in relation to oxygen depletion and its ecological significance in a polluted estuary. Environ. Pollut. 2, 13-35.

Sandbacka, M., Christianson, I., Isomaa, B., 2000. The acute toxicity of surfactants on fish cells, Daphnia magna and fish - a comparative study. Toxicol. in Vitro 14, 61-68.

Selye, H., 1973. The evolution of the stress concept. Am. Sci. 61, 692-699.

Shaffer, P.L., Gold, H.J., 1985. A simulation model of population dynamics of the codling moth, Cydia pomonella. Ecol. Model. 30, 247-274.

Sun, R.Y., Li, B., Zhuge, Y., Shang, Y.C., 1993. General Ecology. Higher Education Press, Beijing. pp. 22-23.

Van Dyk, J.S., Pletschke, B., 2011. Review on the use of enzymes for the detection of organochlorine, organophosphate and carbamate pesticides in the environment. Chemosphere 82 (3), 291-307.

Vesanto, J., Himberg, J., Alhoniemi, E., Parhankangas, J., Team, S., Oy L., 2000. Som toolbox for matlab. Tech. Ber., Helsinki University of Technology.

Wang, Y., Liu, X., Hu, J., Li, L., Li, Y., 2007. The simulation and experimental study of conductance sensor's electric field distribution. Petrol. Instrum. 1 (21), 16-18 (in Chinese).

Ward Jr., J., 1963. Hierarchical grouping to optimize an objective function. J. Am. Stat. Assoc. 58, 236-244.

Wishart, D., 1969. An algorithm for hierarchical classifications. Biometrics 25, 165170 .

Xuereb, B., Lefèvre, E., Garric, J., Geffard, O., 2009. Acetylcholinesterase activity in gammarus fossarum (Crustacea Amphipoda): linking AChE inhibition and behavioural alteration. Aquat. Toxicol. 94, 114-122. 